\title{
Global Dynamics of a Host-Vector-Predator Mathematical Model
}

\author{
Fengyan Zhou ${ }^{1,2}$ and Hongxing Yao ${ }^{1,3}$ \\ ${ }^{1}$ Faculty of Science, Jiangsu University, Zhenjiang, Jiangsu 212013, China \\ ${ }^{2}$ Department of Mathematics, Shaoxing University, Shaoxing, Zhejiang 31200, China \\ ${ }^{3}$ School of Finance and Economics, Jiangsu University, Zhenjiang, Jiangsu 212013, China \\ Correspondence should be addressed to Hongxing Yao; hxyao@ujs.edu.cn
}

Received 18 April 2014; Accepted 2 July 2014; Published 22 July 2014

Academic Editor: Peter G L Leach

Copyright (C) 2014 F. Zhou and H. Yao. This is an open access article distributed under the Creative Commons Attribution License, which permits unrestricted use, distribution, and reproduction in any medium, provided the original work is properly cited.

A mathematical model which links predator-vector(prey) and host-vector theory is proposed to examine the indirect effect of predators on vector-host dynamics. The equilibria and the basic reproduction number $R_{0}$ are obtained. By constructing Lyapunov functional and using LaSalle's invariance principle, global stability of both the disease-free and disease equilibria are obtained. Analytical results show that $R_{0}$ provides threshold conditions on determining the uniform persistence and extinction of the disease, and predator density at any time should keep larger or equal to its equilibrium level for successful disease eradication. Finally, taking the predation rate as parameter, we provide numerical simulations for the impact of predators on vector-host disease control. It is illustrated that predators have a considerable influence on disease suppression by reducing the density of the vector population.

\section{Introduction}

Host-vector diseases are infectious diseases caused by an infectious microbe transmitted by a blood (or sap-) sucking arthropod called vectors, which carry the disease without getting it themselves. For instance, human and animal diseases are such as malaria, dengue fever, West Nile virus, Chagas disease, sleeping sickness, and Lyme disease. Host-vector disease also affects other living organisms, such as plants. Examples of vector-borne infections in crops include tobacco mosaic virus (TMV), tomato spotted wilt virus (TSWV), tomato yellow leaf curl virus (TYLCV), cucumber mosaic virus (CMV), and potato virus Y (PVY) [1]. Vector-borne infections in trees include the pine wilt disease and the red ring disease in palms [2].

Recently, the frequent occurrence of natural disasters and environmental degradation around the world creates conditions suitable for breeding of vectors so that vectorborne diseases are constantly emerging. It is estimated that almost three-fourths of the new infectious diseases in recent years belong to vector-borne infectious disease. Vector-borne diseases remain a serious global threat to humans, livestock, and crops and cause great economic losses in agriculture and forestry; thus; control of such diseases is of great economic and public health concern.

The present mode of controlling vector-borne disease includes using bednets, spraying insecticides [3], and sterile insect technique (SIT) [4-6]. Effective vaccines and treatment control have not been adopted for most of the diseases except that malaria, which is preventable and curable when treatment and prevention measures are taken properly. One potential approach to control vector-borne disease is to introduce biological enemies (biocontrol agents) of the vector. Compared to insecticide method and sterile insect technique, introducing biological enemies (biocontrol agents) of the vector is more safe and cost-efficient. Moreover, it will lead to ecological balance and environment protection. Biological control of vectors has been successfully applied in controlling a variety of disease pathogens, including crop diseases such as the tomato leaf curl virus in India and the cassava mosaic virus in sub-Saharan Africa [7, 8], as well as tree diseases such as pine wilt disease in Japan and China $[9,10]$. For human diseases, Entomogenous fungi are used as promising biopesticides for tick and sleeping sickness control [11, 12]. Predators have been introduced as biological control agents of vectors for various diseases such as malaria, dengue fever, 
tick disease, and Lyme disease [13-20]. Several recent studies suggested that predators led to a decline in local cases of dengue fever in Vietnam and Thailand $[21,22]$ and malaria in India [23, 24].

Mathematical models provide a powerful tool to understand the dynamics of disease spreading through a population and in decision-making process regarding disease prediction and disease control. Since Ross [25] first proposed a malaria model in 1911, many authors have been attracted to mathematical modeling, and very large good results on the subject have been presented. For example, the authors in [26] studied the global dynamics and back bifurcation of a vectorborne disease model with horizontal transmission in host population, which includes exposed classes both in host and vector populations and extended models studied in $[27,28]$.

However, compared with biological control of herbivorous pests, which has long been established as a major component of pest management programmers and is aimed to direct decrease pest densities by pest enemies $[29,30]$, biological control of vectors has been seldom investigated based on mathematical models. The main reason is that modeling the biological control of vectors is a complex interaction, mainly including the virus-host interaction, vector-host interaction, and vector- (prey-) enemy interaction, which is more complicated than the only pest-enemy interaction [31,32] of biological pest control.

Up to know, only several authors have studied the biological control of vector to reduce the disease incidence by mathematical models. For examples, Moore et al. [33] first proposed a host-vector-predator model to study the effect of predator on the transmission and control of vectorborne disease. The efficacy of three types of biocontrol agents: predator/parasitoid, competitor, and pathogen of the vector, is compared in [34] to reduce disease incidence. Zhou and Yao [35] improved the model proposed in [33], study the disease control threshold, and limit cycles with persistence of disease or without disease. However, both papers [33, 35] focus on the constant total host populations, and the hosts and vectors are simply divided into susceptible and infective ones.

Motivated by the above considerations, in this paper we consider a new host-vector-predator model, where both total host and vector population are time-dependent population size and the total host population is divided into four subpopulations of susceptible hosts, exposed hosts, infectious hosts, and recovered hosts. Furthermore, the total vector population is divided into three subpopulations of susceptible hosts, exposed hosts, and infectious hosts. The aim is to explore the global dynamics of the proposed host-vectorpredator model and the impacts of predator on host-vector disease control.

The rest of the paper is organized as follows. In Section 2, we present a formulation of the mathematical model. The equilibria and the basic reproduction number are given in Section 3. In Section 4, global stability analysis of the equilibria is investigated. In Section 5, we use some numerical simulations to explore the impact of predators on the prevalence of vector-borne disease. Conclusions are given in Section 6.

\section{Model Formulation}

The basic model for the transmission dynamics of vectorborne disease with predator control is given by the following deterministic system of nonlinear differential equations:

$$
\begin{gathered}
\frac{d S_{h}(t)}{d t}=b_{1}-\beta_{1} S_{h}(t) I_{v}(t)-\mu_{h} S_{h}(t), \\
\frac{d E_{h}(t)}{d t}=\beta_{1} S_{h}(t) I_{v}(t)-\left(\alpha_{h}+\mu_{h}\right) E_{h}(t), \\
\frac{d I_{h}(t)}{d t}=\alpha_{h} E_{h}(t)-\left(\gamma_{h}+\delta_{h}+\mu_{h}\right) I_{h}(t), \\
\frac{d R_{h}(t)}{d t}=\gamma_{h} I_{h}(t)-\mu_{h} R_{h}(t), \\
\frac{d S_{v}(t)}{d t}=b_{2}-\beta_{2} S_{v}(t) I_{h}(t)-\mu_{v} S_{v}(t)-h S_{v}(t) P_{v}(t), \\
\frac{d E_{v}(t)}{d t}=\beta_{2} S_{v}(t) I_{h}(t)-\alpha_{v} E_{v}(t)-\mu_{v} E_{v}(t)-h E_{v}(t) P_{v}(t), \\
\frac{d I_{v}(t)}{d t}=\alpha_{v} E_{v}(t)-\mu_{v} I_{v}(t)-h I_{v}(t) P_{v}(t), \\
\frac{d P_{v}(t)}{d t}=\varepsilon h\left(S_{v}(t)+E_{v}(t)+I_{v}(t)\right) P_{v}(t)-e P_{v}(t),
\end{gathered}
$$

with initial conditions

$$
\begin{array}{lll}
S_{h}(0) \geq 0, & E_{h}(0) \geq 0, & I_{h}(0) \geq 0, \\
R_{h}(0) \geq 0, & S_{v}(0) \geq 0, & E_{v}(0) \geq 0, \\
I_{v}(0) \geq 0, & P_{v}(0) \geq 0, &
\end{array}
$$

where the total host population at time $t$ denoted by $N_{h}(t)$ is divided into four subpopulations of susceptible hosts $S_{h}(t)$, exposed hosts $E_{h}(t)$, infectious hosts $I_{h}(t)$, and recovered hosts $R_{h}(t)$, so that $N_{h}(v)=S_{h}(t)+E_{h}(t)+I_{h}(t)+R_{h}(t)$. The total vector population at time $t$ denoted by $N_{v}(t)$ is split into susceptible vectors $S_{v}(t)$, exposed vectors $E_{v}(t)$ and infectious vectors $I_{v}(t)$, so that $N_{v}(t)=S_{v}(t)+E_{v}(t)+I_{v}(t)$. The predator of the vector at time $t$ is represented by $P_{v}(t) . b_{1}$ and $b_{2}$ are, respectively, the recruitment rate of hosts and vectors. Parameters $\beta_{1}$ and $\beta_{2}$ are, respectively, the rate of biting from susceptible hosts to infected hosts and susceptible vectors to infected vectors. $\mu_{h}$ and $\mu_{v}$ are, respectively, the natural death rate of infected hosts and vectors. Exposed hosts and vectors develop symptoms of the disease and move to the infectious class at rates $\alpha_{h}$ and $\alpha_{v}$, respectively. $\gamma_{h}$ is the natural recovery rate from the infected hosts. $\delta_{h}$ is the disease-caused death rate of infected hosts. $h$ and $\varepsilon$ are, respectively, the predation rates and conversation rate of the predator to the vector. $e$ is the mortality of the predator including naturally, being preyed both by humans and other animals. 
Adding the host equations in (1), we have

$$
\frac{d N_{h}(t)}{d t}=b_{1}-\mu_{h} N_{h}(t)-\delta_{h} I_{h}(t)
$$

and from the last four equations of system (1), we have

$$
\begin{aligned}
& \frac{d N_{v}(t)}{d t}=b_{2}-\mu_{v} N_{v}(t)-h N_{v}(t) P_{v}(t), \\
& \frac{d P_{v}(t)}{d t}=\varepsilon h N_{v}(t) P_{v}(t)-e P_{v}(t) .
\end{aligned}
$$

By (3) and (4), we have

$$
\begin{aligned}
& \frac{d N_{h}(t)}{d t} \leq b_{1}-\mu_{h} N_{h}(t), \\
& \frac{d N_{v}(t)}{d t} \leq b_{2}-\mu_{v} N_{v}(t), \\
& \frac{d P_{v}(t)}{d t}=\varepsilon h N_{v}(t) P_{v}(t)-e P_{v}(t) .
\end{aligned}
$$

Let

$$
\begin{gathered}
\bar{\Gamma}=\left\{\left(S_{h}, E_{h}, I_{h}, R_{h}, S_{v}, E_{v}, I_{v}, P_{v}\right) \in R_{8}^{+}\right. \\
\mid 0 \leq S_{h}+E_{h}+I_{h}+R_{h} \leq \frac{b_{1}}{\mu_{h}}, \\
\left.0 \leq S_{v}+E_{v}+I_{v} \leq \frac{b_{2}}{\mu_{v}}, P_{v} \geq 0\right\} ;
\end{gathered}
$$

then, it is easy to verify that $\Gamma$ is positively an invariant of system (1).

Since the fourth equation for $R_{h}(t)$ in system (1) does not influence the dynamics behavior of system, we can omit the equations for $R_{h}(t)$. System (1) in the invariant space $\bar{\Gamma}$ can be written as the following seven dimensional nonlinear systems:

$$
\begin{gathered}
\frac{d S_{h}(t)}{d t}=b_{1}-\beta_{1} S_{h}(t) I_{v}(t)-\mu_{h} S_{h}(t), \\
\frac{d E_{h}(t)}{d t}=\beta_{1} S_{h}(t) I_{v}(t)-\left(\alpha_{h}+\mu_{h}\right) E_{h}(t), \\
\frac{d I_{h}(t)}{d t}=\alpha_{h} E_{h}(t)-m I_{h}(t), \\
\frac{d S_{v}(t)}{d t}=b_{2}-\beta_{2} S_{v}(t) I_{h}(t)-\mu_{v} S_{v}(t)-h S_{v}(t) P_{v}(t), \\
\frac{d E_{v}(t)}{d t}=\beta_{2} S_{v}(t) I_{h}(t)-\alpha_{v} E_{v}(t)-\mu_{v} E_{v}(t)-h E_{v}(t) P_{v}(t), \\
\frac{d I_{v}(t)}{d t}=\alpha_{v} E_{v}(t)-\mu_{v} I_{v}(t)-h I_{v}(t) P_{v}(t), \\
\frac{d P_{v}(t)}{d t}=\varepsilon h\left(S_{v}(t)+E_{v}(t)+I_{v}(t)\right) P_{v}(t)-e P_{v}(t),
\end{gathered}
$$

where $m=\gamma_{h}+\delta_{h}+\mu_{h}$. Let

$$
\begin{gathered}
\Gamma=\left\{\left(S_{h}, E_{h}, I_{h}, S_{v}, E_{v}, I_{v}, P_{v}\right) \in R_{7}^{+}\right. \\
\mid 0 \leq S_{h}+E_{h}+I_{h} \leq \frac{b_{1}}{\mu_{h}}, \\
\left.0 \leq S_{v}+E_{v}+I_{v} \leq \frac{b_{2}}{\mu_{v}}, P_{v} \geq 0\right\} .
\end{gathered}
$$

Obviously, for system (7), the region $\Gamma$ is positively invariant.

\section{The Equilibria and the Basic Reproduction Number}

Lemma 1. The equilibria of system (7) are as follows.

(i) If the predator is present, that is, $P_{v}(t)>0$ for any $t \in[0,+\infty)$, then there exist a disease-free equilibrium $E_{1}\left(S_{h}^{0}, 0,0, S_{v}^{0}, 0,0, \widetilde{P}_{v}\right)$ and a disease equilibrium $E_{2}\left(\widetilde{S}_{h}, \widetilde{E}_{h}, \widetilde{I}_{h}, \widetilde{S}_{v}, \widetilde{E}_{v}, \widetilde{I}_{v}, \widetilde{P}_{v}\right)$ if $R_{0}$ is larger than one.

(ii) If the predator is absent, that is, $P_{v}(t) \equiv 0$ for any $t \in[0,+\infty)$, then there exist a disease-free equilibrium $E_{3}\left(\left(b_{1} / \mu_{h}\right) 0,0,\left(b_{2} / \mu_{v}\right), 0,0,0\right)$ and a disease equilibrium $E_{4}\left(\widehat{S}_{h}, \widehat{E}_{h}, \widehat{I}_{h}, \widehat{S}_{v}, \widehat{E}_{v}, \widehat{I}_{v}, 0\right)$ if $R_{1}$ is larger than one.

where

$$
\begin{aligned}
& S_{h}^{0}=\frac{b_{1}}{\mu_{h}}, \quad S_{v}^{0}=\widetilde{N}_{v}, \\
& \widetilde{S}_{h}=\frac{Q_{1} Q_{2} Q_{3}\left(\mu_{v}+h \widetilde{P}_{v}+\beta_{2} \widetilde{I}_{h}\right)}{\alpha_{h} \alpha_{v} \beta_{1} \beta_{2} \widetilde{N}_{v}}, \\
& \widetilde{I}_{h}=\frac{R_{0}^{2}-1}{Q_{1}^{2} Q_{2}^{2} Q_{3} Q_{4} \mu_{h}\left(\mu_{h} \beta_{2} Q_{3}+\alpha_{v} \beta_{1} \beta_{2} \widetilde{N}_{v}\right)}, \\
& \widetilde{E}_{h}=\frac{Q_{2}}{\alpha_{h}} \widetilde{I}_{h}, \\
& \widetilde{E}_{v}=\frac{\alpha_{v} \beta_{2} \widetilde{N}_{v} \widetilde{I}_{h}\left(\mu_{v}+h \widetilde{P}_{v}\right)}{\alpha_{v} Q_{3}\left(\mu_{v}+h \widetilde{P}_{v}+\beta_{2} \widetilde{I}_{h}\right)}, \\
& \widetilde{I}_{v}=\frac{\alpha_{v} \beta_{2} \widetilde{N}_{v} \widetilde{I}_{h}}{Q_{3}\left(\mu_{v}+h \widetilde{P}_{v}+\beta_{2} \widetilde{I}_{h}\right)}, \\
& \widehat{S}_{h}=\frac{Q_{1} Q_{2} \widetilde{I}_{h} \mu_{v}\left(\mu_{v}+\beta_{2} \widehat{I}_{h}\right)}{\alpha_{h} \alpha_{v} \beta_{1} \beta_{2} b_{2}},
\end{aligned}
$$




$$
\begin{aligned}
& \widehat{I}_{h}=\frac{R_{1}^{2}-1}{Q_{1}^{2} Q_{2}^{2} Q_{5} \mu_{h}\left[\mu_{h} \mu_{v} \beta_{2} Q_{5}+\alpha_{v} \beta_{1} \beta_{2} b_{2}\right]}, \\
& \widehat{E}_{h}=\frac{Q_{2}}{\alpha_{h}} \widehat{I}_{h}, \quad \widehat{S}_{v}=\frac{b_{2}}{\mu_{v}+\beta_{2} \widehat{I}_{h}}, \\
& \widehat{E}_{v}=\frac{\mu_{v} \widehat{I}_{v}}{\alpha_{v}}, \quad \widehat{I}_{v}=\frac{b_{2} \alpha_{v} \beta_{2} \widehat{I}_{h}}{\mu_{v} Q_{5}\left(\mu_{v}+\beta_{2} \widehat{I}_{h}\right)}, \\
& Q_{1}=\alpha_{h}+\mu_{h}, \quad Q_{2}=m=\gamma_{h}+\delta_{h}+\mu_{h}, \\
& Q_{3}=\alpha_{v}+\mu_{v}+h \widetilde{P}_{v}, \quad Q_{4}=\left(\mu_{v}+h \widetilde{P}_{v}\right), \\
& Q_{5}=\alpha_{v}+\mu_{v}, \quad \widetilde{N}_{v}=\frac{e}{\varepsilon h}, \\
& \widetilde{P}_{v}=\frac{b_{2}-\mu_{v} \widetilde{N}_{v}}{h \widetilde{N}_{v}} \quad \text { if } \frac{b_{2}}{\mu_{v}}>\frac{e}{\varepsilon h} .
\end{aligned}
$$

Here $\left(b_{2} / \mu_{v}\right)$ is the equilibrium level of total vector population of system (7) without predators, while (e/ch) is the equilibrium level of total vector population of system (7) with predators. $\left(b_{2} / \mu_{v}\right)>(e / \varepsilon h)$ is the sufficient and necessary conditions to ensure $\widetilde{P}_{v}>0$. Therefore, from the viewpoint of biological meaning, if the predators are present, we always assume that $\left(b_{2} / \mu_{v}\right)>(e / \varepsilon h)$.

$R_{0}=\sqrt{\left(\alpha_{h} \alpha_{v} \beta_{1} \beta_{2} b_{1} \widetilde{N}_{v} / \mu_{h} Q_{1} Q_{2} Q_{3} Q_{4}\right)}$ and $R_{1}=$ $\sqrt{\left(\alpha_{h} \alpha_{v} b_{1} b_{2} \beta_{1} \beta_{2} / \mu_{h} \mu_{v}^{2} Q_{1} Q_{2} Q_{5}\right)}$, respectively, are the basic reproduction number of system (7) and system (7) without predators by $[36,37]$.

Remark 2. It is not difficult to find that $R_{0}$ is less than $R_{1}$. That is, predation results into a reduction in the basic reproduction number $R_{0}$ of system (7).

\section{Global Stability Analysis}

The purpose of this section is to discuss the global stability of the disease-free and disease equilibria of system (7) to obtain the control condition under which diseases can be eradicated by predators preying on vectors. Before giving the main proof, we first give the following Lemma.

Lemma 3. For system (4), the unique positive equilibrium $\left(\widetilde{N}_{v}, \widetilde{P}_{v}\right)$ is globally asymptotically stable, where $\widetilde{N}_{v}$ and $\widetilde{P}_{v}$ are given in Lemma 1.

By constructing Lyapunov function $V(t)=$ $(\varepsilon / 2)\left(N_{v}(t)-\widetilde{N}_{v}\right)^{2}+\widetilde{N}_{v}\left(P_{v}(t)-\widetilde{P}_{v}-\widetilde{P}_{v} \ln \left(P_{v}(t) / \widetilde{P}_{v}\right)\right)$ and using LaSalle's invariance principle, it is not difficult to prove that the positive equilibrium $\left(\widetilde{N}_{v}, \widetilde{P}_{v}\right)$ is globally asymptotically stable. Here we omit it.

Theorem 4. If $R_{0} \leq 1$ and for any $t \in[0,+\infty), P_{v}(t) \geq \widetilde{P}_{v}$; then, the disease-free equilibrium $E_{1}$ of system (7) is globally asymptotically stable.
Proof. We construct the following Lyapunov functional:

$$
\begin{aligned}
L(t)= & w_{0}\left(S_{h}(t)-S_{h}^{0}-S_{h}^{0} \ln \frac{S_{h}(t)}{S_{h}^{0}}\right)+w_{1} E_{h}(t) \\
& +w_{2} I_{h}(t)+w_{3}\left(S_{v}(t)-S_{v}^{0}-S_{v}^{0} \ln \frac{S_{v}(t)}{S_{v}^{0}}\right) \\
& +w_{4} E_{v}(t)+w_{5} I_{v}(t) \\
& +w_{6}\left(P_{v}(t)-\widetilde{P}_{v}-\widetilde{P}_{v} \ln \frac{P_{v}(t)}{\widetilde{P}_{v}}\right)
\end{aligned}
$$

where $w_{0}=w_{1}=\left(\alpha_{h} / Q_{1}\right), w_{2}=1, w_{3}=w_{4}=$ $\left(b_{1} \alpha_{h} \alpha_{v} \beta_{1} / \mu_{h} Q_{1} Q_{3} Q_{4}\right), w_{5}=\left(b_{1} \alpha_{h} \beta_{1} / \mu_{h} Q_{1} Q_{4}\right), w_{6}=$ $\left(w_{3} / \varepsilon\right)$.

Calculating the derivative of $L(t)$ along the solution of (7) yields that

$$
\begin{aligned}
\frac{d L(t)}{d t}= & w_{0}\left(\frac{S_{h}(t)-S_{h}^{0}}{S_{h}(t)}\right)\left[b_{1}-\beta_{1} S_{h}(t) I_{v}(t)-\mu_{h} S_{h}(t)\right] \\
& +w_{1}\left[\beta_{1} S_{h}(t) I_{v}(t)-\left(\alpha_{h}+\mu_{h}\right) E_{h}(t)\right] \\
& +w_{2}\left[\alpha_{h} E_{h}(t)-m I_{h}(t)\right] \\
& +w_{3}\left(\frac{S_{v}(t)-S_{v}^{0}}{S_{v}(t)}\right) \\
& \times\left[b_{2}-\beta_{2} S_{v}(t) I_{h}(t)-\mu_{v} S_{v}(t)-h S_{v}(t) P_{v}(t)\right] \\
& +w_{4}\left[\beta_{2} S_{v}(t) I_{h}(t)-\alpha_{v} E_{v}(t)\right. \\
& \left.-\mu_{v} E_{v}(t)-h E_{v}(t) P_{v}(t)\right] \\
& +w_{5}\left[\alpha_{v} E_{v}(t)-\mu_{v} S_{v}(t)-h I_{v}(t) P_{v}(t)\right] \\
& +w_{6}\left(\frac{P_{v}(t)-\widetilde{P}_{v}}{P_{v}(t)}\right) \\
& \times\left[\varepsilon h\left(S_{v}(t)+E_{v}(t)+I_{v}(t)\right)-e\right] P_{v}(t) .
\end{aligned}
$$

By the equilibrium conditions $b_{1}=\mu_{h} S_{h}^{0}$ and $b_{2}=\mu_{v} S_{v}^{0}+$ $h S_{v}^{0} \widetilde{P}_{v}$, it follows that

$$
\begin{aligned}
& \frac{d L(t)}{d t} \\
& \quad-\quad \mu_{h} w_{0} \frac{\left(S_{h}(t)-S_{h}^{0}\right)^{2}}{S_{h}(t)}-\mu_{v} w_{3} \frac{\left(S_{v}(t)-S_{v}^{0}\right)^{2}}{S_{v}(t)} \\
& \quad+\left(w_{1}-w_{0}\right) \beta_{1} S_{h}(t) I_{v}(t)+\left(w_{4}-w_{3}\right) \beta_{2} S_{v}(t) I_{h}(t) \\
& \quad+\left(w_{2} \alpha_{h}-w_{1} Q_{1}\right) E_{h}(t)+\left(w_{5} \alpha_{v}-w_{4} Q_{3}\right) E_{v}(t)
\end{aligned}
$$




$$
\begin{aligned}
& +\left[w_{3} \beta_{2} \widetilde{N}_{v}-w_{2} Q_{2}\right] I_{h}(t)+\left[w_{0} \beta_{1} \frac{b_{1}}{\mu_{h}}-w_{5} Q_{4}\right] I_{v}(t) \\
& +w_{3} h \frac{\left(S_{v}(t)-S_{v}^{0}\right)}{S_{v}(t)}\left(S_{v}^{0} \widetilde{P}_{v}-S_{v}(t) P_{v}(t)\right) \\
& +w_{4} h E_{v}(t)\left(\widetilde{P}_{v}-P_{v}(t)\right) \\
& +w_{5} h I_{v}(t)\left(\widetilde{P}_{v}-P_{v}(t)\right)+w_{6} \varepsilon h I_{v}(t)\left(P_{v}(t)-\widetilde{P}_{v}\right) \\
& \times\left(N_{v}(t)-\widetilde{N}_{v}\right) .
\end{aligned}
$$

Since

$$
\begin{aligned}
w_{3} h & \frac{\left(S_{v}(t)-S_{v}^{0}\right)}{S_{v}(t)}\left(S_{v}^{0} \widetilde{P}_{v}-S_{v}(t) P_{v}(t)\right) \\
& +w_{4} h E_{v}(t)\left(\widetilde{P}_{v}-P_{v}(t)\right) \\
= & -w_{3} h \widetilde{P}_{v} \frac{\left(S_{v}(t)-S_{v}^{0}\right)^{2}}{S_{v}(t)} \\
& +w_{3} h\left(S_{v}(t)-S_{v}^{0}\right)\left(\widetilde{P}_{v}-P_{v}(t)\right),
\end{aligned}
$$

then using $S_{h}^{0}=b_{1} / \mu_{h}, S_{v}^{0}=\widetilde{N}_{v}$ we have

$$
\begin{aligned}
& \frac{d L(t)}{d t} \\
& \quad-\mu_{h} w_{0} \frac{\left(S_{h}(t)-\left(b_{1} / \mu_{h}\right)\right)^{2}}{S_{h}(t)}-w_{3}\left(\mu_{v}+h \widetilde{P}_{v}\right) \frac{\left(S_{v}(t)-\widetilde{N}_{v}\right)^{2}}{S_{v}(t)} \\
& \quad+Q_{2}\left(R_{0}^{2}-1\right) I_{h}(t)+\left[w_{3} h\left(S_{v}(t)-\widetilde{N}_{v}\right)\right. \\
& \left.\quad+w_{4} h E_{v}(t)+w_{5} h I_{v}(t)\right] \\
& \quad \times\left(\widetilde{P}_{v}-P_{v}(t)\right) \quad+w_{6} \operatorname{sh}\left(P_{v}(t)-\widetilde{P}_{v}\right)\left(N_{v}(t)-\widetilde{N}_{v}\right) .
\end{aligned}
$$

After some rearrangement, we have

$$
\begin{aligned}
& \frac{d L(t)}{d t} \\
& =-\mu_{h} w_{0} \frac{\left(S_{h}(t)-\left(b_{1} / \mu_{h}\right)\right)^{2}}{S_{h}(t)}-w_{3}\left(\mu_{v}+h \widetilde{P}_{v}\right) \frac{\left(S_{v}(t)-\widetilde{N}_{v}\right)^{2}}{S_{v}(t)} \\
& \quad+Q_{2}\left(R_{0}^{2}-1\right) I_{h}(t)+h\left(w_{5}-w_{3}\right) I_{v}(t)\left(\widetilde{P}_{v}-P_{v}\right) .
\end{aligned}
$$

Since $P_{v}(t) \geq \widetilde{P}_{v}$, then we have $N_{v}(t) \leq \widetilde{N}_{v}$ for any $t \in$ $[0,+\infty)$. Otherwise, if $N_{v}(t)>\widetilde{N}_{v}$ for any $t \in[0,+\infty)$, then $\varepsilon h N_{v}(t)-e>0$; thus, from the last equation of system (7), we have $\left(d P_{v}(t) / d t\right)>0$. On the other hand, by Lemma 1 , $\lim _{t \rightarrow+\infty} P_{v}(t)=\widetilde{P}_{v}$, so for any $t \in[0,+\infty), P_{v}(t)<\widetilde{P}_{v}$. This contradicts $P_{v}(t) \geq \widetilde{P}_{v}$. So $N_{v}(t) \leq \widetilde{N}_{v}$ when $P_{v}(t) \geq \widetilde{P}_{v}$ for any $t \in[0,+\infty)$. Thus, $(d L(t) / d t) \leq 0$ if $R_{0} \leq 1$ and $P_{v}(t) \geq \widetilde{P}_{v}$ for any $t \in[0,+\infty)$. Furthermore, $(d L(t) / d t)=0$ if and only if $S_{h}=S_{h}^{0}, S_{v}=S_{v}^{0}=\widetilde{N}_{v}, E_{h}=E_{v}=I_{h}=I_{v}=0$ and $P_{v}=\widetilde{P}_{v}$. Consequently, the largest compact invariant set in $\left\{\left(S_{h}(t), E_{h}(t), I_{h}(t), S_{v}(t), E_{v}(t), I_{v}(t), P_{v}(t)\right) \in \Gamma: d L / d t=0\right\}$ when $R_{0} \leq 1$ is the singleton $E_{1}$. Then by Lyapunov-LaSalle theorem, the equilibrium $E_{1}$ is globally stable if $R_{0} \leq 1$ and $P_{v}(t) \geq \widetilde{P}_{v}$ for any $t \in[0,+\infty)$.

Remark 5. From Theorem 4 we can see that when $R_{0} \leq$ 1 the disease-free equilibrium $E_{1}$ of system (7) is globally asymptotically stable if the predator population size $P_{v}(t)$ is not less than the predator equilibrium level $\widetilde{P}_{v}$, which depends on the total equilibrium vector density $\widetilde{N}_{v}$. That is, the predator density threshold for successful disease eradication is $P_{v}(t)=\widetilde{P}_{v}$. If $P_{v}(t) \geq \widetilde{P}_{v}$ and $R_{0} \leq 1$ then disease can be eradicated; otherwise; pathogen persists though predators are introduced and $R_{0} \leq 1$.

Similar to the proof of Theorem 4, we have the following corollary to show that the disease-free equilibrium $E_{3}$ of system (7) in absence of predators is global asymptotically stable.

Corollary 6. If $R_{1} \leq 1$ then the disease-free equilibrium $E_{3}$ of system (7) without predators is globally asymptotically stable.

A global stability result for the endemic equilibrium $E_{2}$ of the system (7) is given below.

Theorem 7. The endemic equilibrium state $E_{2}$ of system (7) is globally asymptotically stable if

$$
R_{0}>1, \quad \alpha_{v}=\frac{\beta_{2} \widetilde{S}_{v} \widetilde{I}_{h}}{\widetilde{E}_{v}}
$$

where $\widetilde{S}_{v}, \widetilde{I}_{h}$ and $\widetilde{E}_{v}$ are the disease equilibrium value of susceptible vectors, infected hosts, and exposed vectors of system (7).

Proof. Consider the following Lyapunov functional:

$V(t)$

$$
\begin{aligned}
= & c_{1}\left(S_{h}(t)-\widetilde{S}_{h} \ln \frac{S_{h}(t)}{\widetilde{S}_{h}}\right)+c_{2}\left(E_{h}(t)-\widetilde{E}_{h} \ln \frac{E_{h}(t)}{\widetilde{E}_{h}}\right) \\
& +c_{3}\left(I_{h}(t)-\widetilde{I}_{h} \ln \frac{I_{h}(t)}{\widetilde{I}_{h}}\right)+c_{4}\left(S_{v}(t)-\widetilde{S}_{v} \ln \frac{S_{v}(t)}{\widetilde{S}_{v}}\right) \\
& +c_{5}\left(E_{v}(t)-\widetilde{E}_{v} \ln \frac{E_{v}(t)}{\widetilde{E}_{v}}\right)+c_{6}\left(I_{v}(t)-\widetilde{I}_{v} \ln \frac{I_{v}(t)}{\widetilde{I}_{v}}\right) \\
& +c_{7}\left(P_{v}(t)-\widetilde{P}_{v} \ln \frac{P_{v}(t)}{\widetilde{P}_{v}}\right),
\end{aligned}
$$


where $c_{2}=c_{1}, c_{3}=\left(c_{1} \beta_{1} a_{1} / d_{1}\right), c_{4}=c_{5}=\left(c_{1} \beta_{1} a_{1} / \beta_{2} a_{2}\right)$, $c_{6}=\left(c_{5} \beta_{2} a_{2} / d_{2}\right), c_{7}=\left(c_{4} / \varepsilon\right), \widetilde{S}_{h} \widetilde{I}_{v}=a_{1}, \widetilde{S}_{v} \widetilde{I}_{h}=a_{2}, d_{1}=$ $\alpha_{h} \widetilde{E}_{h}, d_{2}=\alpha_{v} \widetilde{E}_{v}$.

Calculate the derivative of $V(t)$ along the solution of (1); this yields that

$$
\begin{aligned}
& \frac{d V(t)}{d t} \\
& =c_{1}\left(1-\frac{\widetilde{S}_{h}}{S_{h}(t)}\right)\left[b_{1}-\beta_{1} S_{h}(t) I_{v}(t)-\mu_{h} S_{h}(t)\right] \\
& \quad+c_{2}\left(1-\frac{\widetilde{E}_{h}}{E_{h}(t)}\right)\left[\beta_{1} S_{h}(t) I_{v}(t)-\left(\alpha_{h}+\mu_{h}\right) E_{h}(t)\right] \\
& \quad+c_{3}\left(1-\frac{\widetilde{I}_{h}}{I_{h}(t)}\right)\left[\alpha_{h} E_{h}(t)-m I_{h}(t)\right] \\
& \quad+c_{4}\left(1-\frac{\widetilde{S}_{v}}{S_{v}(t)}\right) \\
& \quad \times\left[b_{2}-\beta_{2} S_{v}(t) I_{h}(t)-\mu_{v} S_{v}(t)-h S_{v}(t) P_{v}(t)\right] \\
& +c_{5}\left(1-\frac{\widetilde{E}_{v}}{E_{v}(t)}\right) \\
& \quad \times\left[\beta_{2} S_{v}(t) I_{h}(t)-\alpha_{v} E_{v}(t)-\mu_{v} E_{v}(t)-h E_{v}(t) P_{v}(t)\right] \\
& +c_{6}\left(1-\frac{\widetilde{I}_{v}}{I_{v}(t)}\right) \\
& \quad \times\left[\alpha_{v} E_{v}(t)-\mu_{v} I_{v}(t)-h I_{v}(t) P_{v}(t)\right] \\
& +c_{7}\left(1-\frac{\widetilde{P}_{v}}{P_{v}(t)}\right) \\
& \times\left[\varepsilon h\left(S_{v}(t)+E_{v}(t)+I_{v}(t)\right) P_{v}(t)-e P_{v}(t)\right] .
\end{aligned}
$$

System (7) satisfied the following relations at equilibrium point:

$$
\begin{aligned}
& b_{1}=\beta_{1} \widetilde{S}_{h} \widetilde{I}_{v}+\mu_{h} \widetilde{S}_{h}, \\
& \left(\alpha_{h}+\mu_{h}\right)=\frac{\beta_{1} \widetilde{S}_{h} \widetilde{I}_{v}}{\widetilde{E}_{h}}, \\
& m=\frac{\alpha_{h} \widetilde{E}_{h}}{\widetilde{I}_{h}}, \\
& b_{2}=\beta_{2} \widetilde{S}_{v} \widetilde{I}_{h}+\mu_{v} \widetilde{S}_{v}+h \widetilde{S}_{v} \widetilde{P}_{v}, \\
& \left(\alpha_{v}+\mu_{v}\right)=\frac{\beta_{2} \widetilde{S}_{v} \widetilde{I}_{h}}{\widetilde{E}_{v}}-h \widetilde{P}_{v}, \\
& \mu_{v}=\frac{\alpha_{v} \widetilde{E}_{v}}{\widetilde{I}_{v}}-h \widetilde{P}_{v}, \\
& e=\varepsilon h \widetilde{N}_{v}, \quad \widetilde{N}_{v}=\widetilde{S}_{v}+\widetilde{E}_{v}+\widetilde{I}_{v} .
\end{aligned}
$$

Substituting $b_{1}$ up to $e$ in the above equation, we obtain $\frac{d V(t)}{d t}$

$$
\begin{aligned}
= & -c_{1} \mu_{h} \frac{\left(S_{h}(t)-\widetilde{S}_{h}\right)^{2}}{S_{h}(t)}-c_{4} \mu_{v} \frac{\left(S_{v}(t)-\widetilde{S}_{v}\right)^{2}}{S_{v}(t)} \\
& +c_{1}\left(1-\frac{\widetilde{S}_{h}}{S_{h}(t)}\right)\left[\beta_{1} \widetilde{S}_{h} \widetilde{I}_{v}-\beta_{1} S_{h}(t) I_{v}(t)\right] \\
& +c_{2}\left(1-\frac{\widetilde{E}_{h}}{E_{h}(t)}\right)\left[\beta_{1} S_{h}(t) I_{v}(t)-E_{h} \frac{\beta_{1} \widetilde{S}_{h} \widetilde{I}_{v}}{\widetilde{E}_{h}}\right] \\
& +c_{3}\left(1-\frac{\widetilde{I}_{h}}{I_{h}(t)}\right)\left[\alpha_{h} E_{h}(t)-\alpha_{h} \widetilde{E}_{h} \frac{I_{h}(t)}{\widetilde{I}_{h}}\right] \\
& +c_{4}\left(1-\frac{\widetilde{S}_{v}}{S_{v}(t)}\right) \\
& \times\left[\beta_{2} \widetilde{S}_{v} \widetilde{I}_{h}-\beta_{2} S_{v}(t) I_{h}(t)+h \widetilde{S}_{v} \widetilde{P}_{v}-h S_{v}(t) P_{v}(t)\right] \\
& +c_{5}\left(1-\frac{\widetilde{E}_{v}}{E_{v}(t)}\right) \\
& \times\left[\beta_{2} S_{v}(t) I_{h}(t)-\beta_{2} \widetilde{S}_{v} \widetilde{I}_{h} \frac{E_{v}(t)}{\widetilde{E}_{v}}\right. \\
& +c_{7}\left(1-\frac{\widetilde{P}_{v}}{P_{v}(t)}\right) \\
& \left.+h \varepsilon_{v}\left(S_{v}(t)+E_{v}(t)+I_{v}(t)-\widetilde{S}_{v}-\widetilde{E}_{v}-\widetilde{I}_{v}\right)\right] P_{v}(t) \\
& +c_{6}\left(1-\frac{\widetilde{I}_{v}}{I_{v}(t)}\right) \\
& {\left[\alpha_{v} E_{v}(t)-\alpha_{v} \widetilde{E}_{v} \frac{I_{v}}{\widetilde{I}_{v}(t)}-h I_{v}(t) P_{v}(t)+h E_{v}(t) \widetilde{P}_{v}\right] } \\
& \left.-\widetilde{P}_{v}\right]
\end{aligned}
$$

Set

$$
\begin{array}{lll}
\frac{S_{h}}{\widetilde{S}_{h}}=x_{1}, & \frac{E_{h}}{\widetilde{E}_{h}}=x_{2}, & \frac{I_{h}}{\widetilde{I}_{h}}=x_{3}, \\
\frac{S_{v}}{\widetilde{S}_{v}}=x_{4}, & \frac{E_{v}}{\widetilde{E}_{v}}=x_{5}, & \frac{I_{v}}{\widetilde{I}_{v}}=x_{6}, \\
\frac{P_{v}}{\widetilde{P}_{v}}=x_{7}, & \widetilde{S}_{v} \widetilde{P}_{v}=a_{3}, & \widetilde{E}_{v} \widetilde{P}_{v}=a_{4}, \\
\widetilde{I}_{v} \widetilde{P}_{v}=a_{5} ; &
\end{array}
$$


then, from the above equation, we obtain

$$
\begin{aligned}
\frac{d V(t)}{d t} & \\
= & -c_{1} \mu_{h} \frac{\left(S_{h}(t)-\widetilde{S}_{h}\right)^{2}}{S_{h}(t)}-c_{4} \mu_{v} \frac{\left(S_{v}(t)-\widetilde{S}_{v}\right)^{2}}{S_{v}(t)} \\
& +c_{1} \beta_{1} a_{1}-c_{1} \beta_{1} a_{1} x_{1} x_{6}-c_{1} \beta_{1} a_{1} \frac{1}{x_{1}}+c_{1} \beta_{1} a_{1} x_{6} \\
& +c_{2} \beta_{1} a_{1}+c_{2} \beta_{1} a_{1} x_{1} x_{6}-c_{2} \beta_{1} a_{1} x_{2}-c_{2} \beta_{1} a_{1} \frac{x_{1} x_{6}}{x_{2}} \\
& +c_{3} d_{1}+c_{3} d_{1} x_{2}-c_{3} d_{1} x_{3}-c_{3} d_{1} \frac{x_{2}}{x_{3}} \\
& +c_{4} \beta_{2} a_{2}+c_{4} \beta_{2} a_{2} x_{3}-c_{4} \beta_{2} a_{2} x_{3} x_{4}-c_{4} \beta_{2} a_{2} \frac{1}{x_{4}} \\
& +c_{4} h a_{3}-c_{4} h a_{3} x_{4} x_{7}-c_{4} h a_{3} \frac{1}{x_{4}}+c_{4} h a_{3} x_{7} \\
& +c_{7} \varepsilon h a_{3}+c_{7} \varepsilon h a_{4}+c_{7} \varepsilon h a_{5} . \\
& +c_{5} \beta_{2} a_{2}+c_{5} \beta_{2} a_{2} x_{3} x_{4}-c_{5} \beta_{2} a_{2} x_{5}-c_{5} \beta_{2} a_{2} \frac{x_{3} x_{4}}{x_{5}} \\
& +c_{6} k h a_{5} x_{6} x_{7}+c_{6} h a_{5} x_{6}+c_{6} h a_{5} x_{7}-c_{6} h a_{5} x_{4} x_{7}+c_{7} \varepsilon h a_{4} x_{5} x_{7}+c_{7} \varepsilon h a_{5} x_{6} x_{7} \\
& +c_{5} h c_{4} x_{5} x_{7}+c_{5} h a_{4} x_{5}+c_{5} h a_{4} x_{7}-c_{5} h a_{4} \\
& +c_{6} d_{2}+c_{6} d_{2} x_{5}-c_{6} d_{2} \frac{x_{5}}{x_{6}}-c_{6} d_{2} x_{6} x_{7} \\
& +c_{7} \varepsilon h a_{4} x_{5}-c_{7} \varepsilon h a_{5} x_{6} \\
& \\
&
\end{aligned}
$$

After some rearrangement, we have

$$
\begin{aligned}
& \frac{d V(t)}{d t} \\
& \quad-\quad c_{1} \mu_{h} \frac{\left(S_{h}(t)-\widetilde{S}_{h}\right)^{2}}{S_{h}(t)}-c_{5} \mu_{v} \frac{\left(S_{v}(t)-\widetilde{S}_{v}\right)^{2}}{S_{v}(t)} \\
& \quad+\left(c_{1} \beta_{1} a_{1}-c_{6} d_{2}\right) x_{6}+\left(c_{3} d_{1}-c_{2} \beta_{1} a_{1}\right) x_{2} \\
& \quad+\left(c_{4} \beta_{2} a_{2}-c_{3} d_{1}\right) x_{3}+\left(c_{6} d_{2}-c_{5} \beta_{2} a_{2}\right) x_{5} \\
& \quad+\left(c_{2} \beta_{1} a_{1}-c_{1} \beta_{1} a_{1}\right) x_{1} x_{6}+\left(c_{5} \beta_{2} a_{2}-c_{4} \beta_{2} a_{2}\right) x_{3} x_{4} \\
& \quad+\left(c_{7} \varepsilon-c_{4}\right) h a_{3} x_{4} x_{7}+\left(c_{7} \varepsilon-c_{5}\right) h a_{4} x_{5} x_{7} \\
& \quad+\left(c_{7} \varepsilon-c_{6}\right) h a_{5} x_{6} x_{7}
\end{aligned}
$$

$$
\begin{aligned}
& +\left(c_{4} a_{3}+c_{5} a_{4}+c_{6} a_{5}-c_{7} \varepsilon a_{3}-c_{7} \varepsilon a_{4}-c_{7} \varepsilon a_{5}\right) h x_{7} \\
& +\left(c_{7} \varepsilon h a_{4}+c_{7} \varepsilon h a_{5}-c_{5} h a_{4}-c_{6} h a_{5}\right) \\
& +\left(c_{4} h a_{3}+c_{7} \varepsilon h a_{3}-c_{4} h a_{3} \frac{1}{x_{4}}-c_{7} \varepsilon h a_{3} x_{4}\right) \\
& +c_{1} \beta_{1} a_{1}-c_{1} \beta_{1} a_{1} \frac{1}{x_{1}}-c_{2} \beta_{1} a_{1} \frac{x_{1} x_{6}}{x_{2}}+c_{2} \beta_{1} a_{1} \\
& -c_{3} d_{1} \frac{x_{2}}{x_{3}}+c_{3} d_{1}+c_{4} \beta_{2} a_{2}-c_{4} \beta_{2} a_{2} \frac{1}{x_{4}} \\
& -c_{5} \beta_{2} a_{2} \frac{x_{3} x_{4}}{x_{5}}+c_{5} \beta_{2} a_{2}-c_{6} d_{2} \frac{x_{5}}{x_{6}}+c_{6} d_{2}
\end{aligned}
$$

By some reduction, it follows that

$$
\begin{aligned}
& \frac{d V(t)}{d t} \\
& \quad-c_{1} \mu_{h} \frac{\left(S_{h}(t)-\widetilde{S}_{h}\right)^{2}}{S_{h}(t)}-c_{5} \mu_{v} \frac{\left(S_{v}(t)-\widetilde{S}_{v}\right)^{2}}{S_{v}(t)} \\
& \quad+c_{4} h a_{3}\left(2-x_{4}-\frac{1}{x_{4}}\right) \\
& \quad+c_{1} \beta_{1} a_{1}\left(6-\frac{1}{x_{1}}-\frac{x_{1} x_{6}}{x_{2}}-\frac{x_{2}}{x_{3}}-\frac{1}{x_{4}}-\frac{x_{3} x_{4}}{x_{5}}-\frac{x_{5}}{x_{6}}\right) .
\end{aligned}
$$

Since the arithmetic mean is greater than or is equal to the geometric mean, then

$$
\begin{aligned}
& 2-x_{4}-\frac{1}{x_{4}} \geq 0, \\
& \frac{1}{x_{1}}+\frac{x_{1} x_{6}}{x_{2}}+\frac{x_{2}}{x_{3}}+\frac{1}{x_{4}}+\frac{x_{3} x_{4}}{x_{5}}+\frac{x_{5}}{x_{6}} \geq 6 .
\end{aligned}
$$

Thus, it follows from (24) that $(d V(t) / d t) \leq 0$ in $\Omega$. The equation $(d V(t) / d t)=0$ holds if and only if $x_{1}=x_{2}=x_{3}=$ $x_{4}=x_{5}=x_{6}=1$; that is, $S_{h}=\widetilde{S}_{h}, E_{h}=\widetilde{E}_{h}, I_{h}=\widetilde{I}_{h}, S_{v}=$ $\widetilde{S}_{v}, E_{v}=\widetilde{E}_{v}, I_{v}=\widetilde{I}_{v}, P_{v}=\widetilde{P}_{v}$. Therefore, we prove the global stability of the disease in $\Gamma$. The maximal compact invariant set in $\left\{S_{h}, E_{h}, I_{h}, S_{v}, E_{v}, I_{v}, P_{v} \in \Gamma: d V(t) / d t=0\right\}$ is $\left\{E_{2}\right\}$ when $R_{0}>1$ and $\alpha_{v}=\left(\beta_{3} \widetilde{S}_{v} \widetilde{I}_{h} / \widetilde{E}_{v}\right)$. From the LaSalle's invariance principle, we finish the proof of Theorem 7 .

Similar to the proof of Theorem 7, we have the following corollary to show that the disease equilibrium $E_{4}$ of system (7) in absence of predators is globally asymptotically stable.

Corollary 8. The endemic equilibrium state $E_{4}$ of system (7) without predators is globally asymptotically stable if $R_{1}>1$.

Remark 9. From Theorems 4 and Theorem 7, Corollary 6 and Corollary 8 we find that $R_{0}=1$ and $R_{1}=1$ provide threshold conditions on determining the uniform persistence and extinction of the disease with and without 


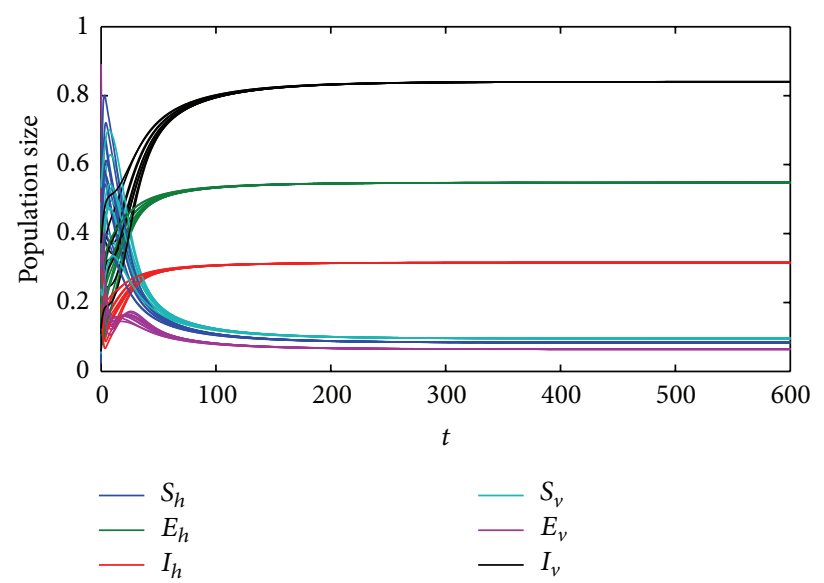

FIGURE 1: Global asymptotically stability of the unique disease equilibrium of system (7) without predators when $R_{1}>1$.

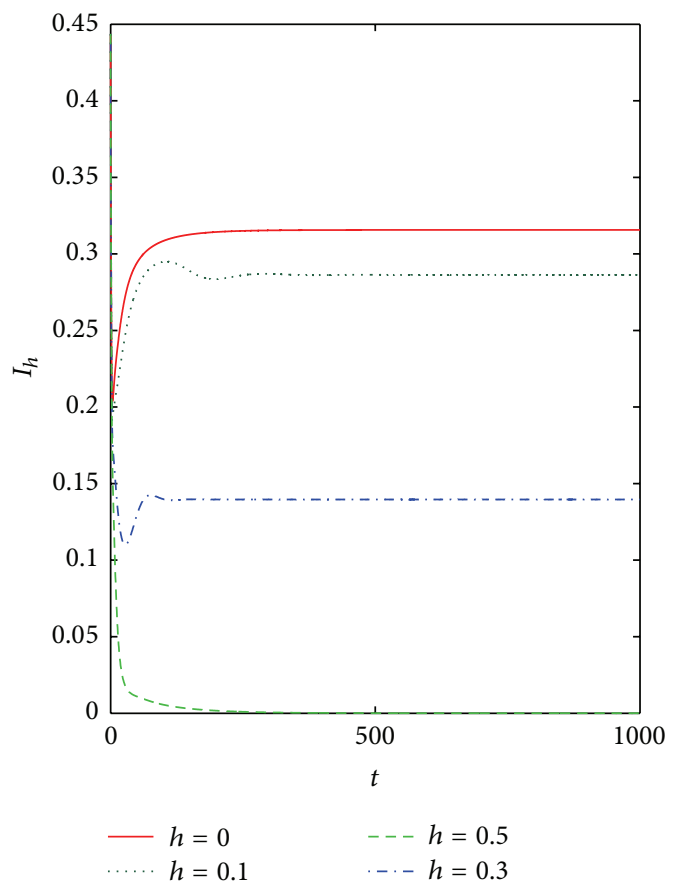

(a)

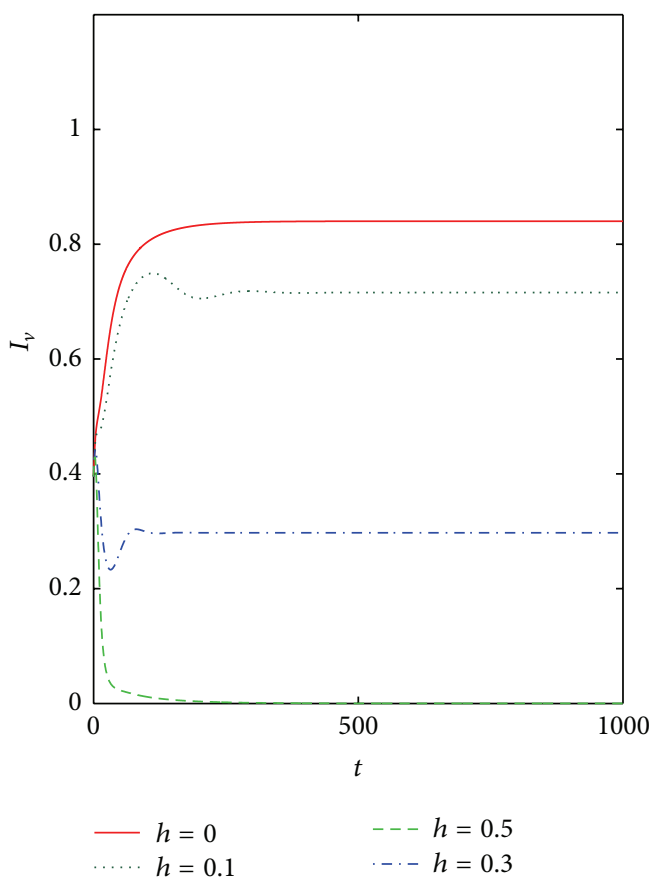

(b)

FIGURE 2: The population densities of the infected hosts and vectors of system (7) without and with predators.

predators, respectively. Moreover, we also find that the disease is persistent without predators if $R_{1}>1$, then by introducing predators, the disease will tend to be extinct if $R_{0} \leq 1$.

\section{Numerical Simulations}

In this section, we examine the effects of predators on the transmission dynamics of vector-borne diseases by some numerical simulations. We choose the following set of parameter values (it should be stated that these parameters are chosen for illustrative purpose only and may not necessarily be realistic epidemiologically): $b_{1}=0.786098, b_{2}=0.190028$, $\beta_{1}=0.961509, \beta_{2}=0.535061, \mu_{h}=0.914518, \mu_{v}=$ $0.0150508, \alpha_{h}=0.645664, \alpha_{v}=0.19636$, and $m=1.120469$; By simple calculations, the basic reproduction number $R_{1}=$ $11.2826>1$; then, by Corollary 8 , the disease equilibrium of system (7) without predators is globally asymptotically stable. That is, the disease persists without predators (see Figure 1).

Choose $e=0.1, \varepsilon=0.15$; we consider the effect of predators on disease control by comparing equilibrium level of infected hosts and vectors with different values of $h$ in absence of the predators, that is, $h=0$; then, by the Corollary 8 disease persists (see the solid line of Figure 2). By introducing predators of the vector population, we find that the equilibrium infection levels have been reduced a bit if the 
predation rate $h$ is equal to 0.1 ; however, the disease persists (see the dotted line of Figure 2). Increasing the predation rate such that $h=0.3$, we find that though the disease still persists, the equilibrium levels of infected hosts and vectors have been greatly lessened (see the dot and dashed line of Figure 2). By enhancing the predation rate $h$ such that $h=$ $0.5>h^{*}=0.4755\left(h^{*}\right.$ satisfies that $\left.R_{0}=1\right)$, then $R_{0}=$ $0.9411<1$; therefore, by Theorem 4 the vector-borne disease can be eradicated by introducing vector predators (see the dashed line of Figure 2).

Remark 10. From Figures 1 and 2, we find that predation has a positive effect on vector-host disease control by reducing vector density. Furthermore, disease can be eradicated if predation rate $h$ is large enough such that $R_{0}(h) \leq 1$ and the predator density $P_{v}(t)$ satisfies $P_{v}(t) \geq \widetilde{P}_{v}$, where $\widetilde{P}_{v}$ is the predator equilibrium level.

\section{Conclusions}

In this paper, we propose a host-vector-predator coupled model with variable host and vector population size to investigate the effect of predators on vector-borne disease control by analyzing the global stability of the disease-free and disease equilibria. It is shown that the basic reproduction number $R_{0}$ characterizes the disease transmission dynamics: if $R_{0} \leq 1$, then there exists only the disease-free equilibrium which is globally asymptotically stable when predator density at any time keeps larger or equal to its equilibrium level; that is, disease tends to be extinct when predators are introduced, and if $R_{0}>1$, then there is a disease equilibrium which is globally asymptotically stable; that is, disease still persists though predators are introduced. As corollaries, the globally stability of system (7) without predators is given. To examine the effect of predator on disease control, numerical simulations are given by choosing to focus on parameter $h$, which is predation rate. We conclude that predation leads to decrease of equilibrium levels both for infected host and vector population; as $h$ increases, then the infected host and vector equilibrium population will be lessened. Furthermore, if $h>h^{*}\left(h^{*}\right.$ satisfies that $\left.R_{0}=1\right)$, then vector-borne diseases can be eradicated.

\section{Conflict of Interests}

The authors declare that there is no conflict of interests regarding the publication of this paper.

\section{Acknowledgments}

The work is supported by the National Natural Science Foundation of China (Grant nos. 70871056, 70871056, 71271103, and 71371087), the National Research Subject of Education Information Technology (Grant no. 126240641), and the Innovative Foundation for Doctoral Candidate of Jiangsu Province, China (Grant no. CXZZ13_0687).

\section{References}

[1] K. G. Scholthof, S. Adkins, H. Czosnek et al., “Top 10 plant viruses in molecular plant pathology," Molecular Plant Pathology, vol. 12, no. 9, pp. 938-954, 2011.

[2] R. M. Giblin-Davis, Interactions of Nematodes with Insects, Nematode Interactions, Chapman and Hall, London, UK, 1993.

[3] J. Hemingway and H. Ranson, "Insecticide resistance in insect vectors of human disease," Annual Review of Entomology, vol. 45, pp. 371-391, 2000.

[4] D. J. Gubler, "Dengue and dengue hemorrhagic fever," Clinical Microbiology Reviews, vol. 11, no. 3, pp. 480-496, 1998.

[5] A. M. Handler and A. A. James, Insect Transgenesis: Methods and Applications, CRC Press, Boca Raton, Fla, USA, 2000.

[6] C. J. McMeniman, R. V. Lane, B. N. Cass et al., "Stable introduction of a life-shortening Wolbachia infection into the mosquito Aedes aegypti," Science, vol. 323, no. 5910, pp. 141-144, 2009.

[7] M. J. Jeger, J. Holt, F. van den Bosch, and L. V. Madden, "Epidemiology of insect-transmitted plant viruses: Modelling disease dynamics and control interventions," Physiological Entomology, vol. 29, no. 3, pp. 291-304, 2004.

[8] M. Otim, J. Legg, S. Kyamanywa, A. Polaszek, and D. Gerling, "Population dynamics of Bemisia tabaci (Homoptera: Aleyrodidae) parasitoids on cassava mosaic disease-resistant and susceptible varieties," Biocontrol Science and Technology, vol. 16, no. 2, pp. 205-214, 2006.

[9] L. Zhang, J. Liu, and H. Wu, "The screening virulent strain of Beauveria bassiana to Monochamus alternatus," Journal of Nanjing Forestry Unversity, vol. 24, pp. 33-37, 2000.

[10] Y. X. Lai, J. D. Liu, Q. Y. Xu, Y. H. Wang, and C. M. Zhou, “Trials on the parasitism of Beauveria bassiana or Verticillium lecanii on larvae of Monochamus alternatus Hope," Journal of Jiangsu University of Science and Technology, vol. 30, no. 4, pp. 7-9, 2003.

[11] G. P. Kaaya and S. Hassan, "Entomogenous fungi as promising biopesticides for tick control," Experimental and Applied Acarology, vol. 24, no. 12, pp. 913-926, 2000.

[12] E.-J. Scholte, K. Ng'Habi, J. Kihonda et al., "An entomopathogenic fungus for control of adult African malaria mosquitoes," Science, vol. 308, no. 5728, pp. 1641-1642, 2005.

[13] D. W. Jenkins, "Pathogens, parasites and predators of medically important arthropods. Annotated list and bibliography," Bulletin of the World Health Organization, vol. 30, pp. 1-150, 1964.

[14] E. Legner, "Biological control of diptera of medical and veterinary importance," Journal of Vector Ecology, vol. 20, pp. 59-120, 1995.

[15] J. R. Stauffer Jr., M. E. Arnegard, M. Cetron et al., "Controlling vectors and hosts of parasitic diseases using fishes," BioScience, vol. 47, no. 1, pp. 41-49, 1997.

[16] M. Samish and J. Rehacek, "Pathogens and predators of ticks and their potential in biological control," Annual Review of Entomology, vol. 44, pp. 159-182, 1999.

[17] R. Kumar and J. S. Hwang, "Larvicidal efficiency of aquatic predators: a perspective for mosquito biocontrol," Zoological Studies, vol. 45, no. 4, pp. 447-466, 2006.

[18] R. S. Ostfeld, A. Price, V. L. Hornbostel, M. A. Benjamin, and F. Keesing, "Controlling ticks and tick-borne zoonoses with biological and chemical agents," BioScience, vol. 56, no. 5, pp. 383-394, 2006.

[19] K. Walker and M. Lynch, "Contributions of Anopheles larval control to malaria suppression in tropical Africa: review of 
achievements and potential," Medical and Veterinary Entomology, vol. 21, no. 1, pp. 2-21, 2007.

[20] X. J. Nelson and R. R. Jackson, "A predator from East Africa that chooses malaria vectors as preferred prey," PLOS ONE, vol. 1, no. 1, article e132, 2006.

[21] B. Kay and V. S. Nam, "New strategy against Aedes aegypti in Vietnam," The Lancet, vol. 365, no. 9459, pp. 613-617, 2005.

[22] P. Kittayapong, S. Yoksan, U. Chansang, C. Chansang, and A. Bhumiratana, "Suppression of dengue transmission by application of integrated vector control strategies at sero-positive GIS-based foci," The American Journal of Tropical Medicine and Hygiene, vol. 78, no. 1, pp. 70-76, 2008.

[23] S. K. Ghosh, S. N. Tiwari, T. S. Sathyanarayan et al., "Larvivorous fish in wells target the malaria vector sibling species of the Anopheles culicifacies complex in villages in Karnataka, India," Transactions of the Royal Society of Tropical Medicine and Hygiene, vol. 99, no. 2, pp. 101-105, 2005.

[24] S. K. Ghosh and A. P. Dash, "Larvivorous fish against malaria vectors: a new outlook," Transactions of the Royal Society of Tropical Medicine and Hygiene, vol. 101, no. 11, pp. 1063-1064, 2007.

[25] R. Ross, "An application of the theory of probabilities to the study of a priori pathometry," Proceedings of the Royal Society of London Series A, vol. 92, no. 638, pp. 204-230, 1916.

[26] A. A. Lashari and G. Zaman, "Global dynamics of vector-borne diseases with horizontal transmission in host population," Computers \& Mathematics with Applications, vol. 61, no. 4, pp. 745-754, 2011.

[27] H. Wei, X. Li, and M. Martcheva, "An epidemic model of a vector-borne disease with direct transmission and time delay," Journal of Mathematical Analysis and Applications, vol. 342, no. 2, pp. 895-908, 2008.

[28] L. Cai and X. Li, "Analysis of a simple vector-host epidemic model with direct transmission," Discrete Dynamics in Nature and Society, vol. 2010, Article ID 679613, 12 pages, 2010.

[29] R. F. Luck, B. M. Shepard, and P. E. Kenmore, "Experimental methods for evaluating arthropod natural enemies," Annual Review of Entomology, vol. 33, pp. 367-389, 1988.

[30] G. Zehnder, G. M. Gurr, S. Kühne, M. R. Wade, S. D. Wratten, and E. Wyss, "Arthropod pest management in organic crops," Annual Review of Entomology, vol. 52, pp. 57-80, 2007.

[31] P. D. N. Srinivasu and B. S. R. Prasad, "Role of quantity of additional food to predators as a control in predator-prey systems with relevance to pest management and biological conservation," Bulletin of Mathematical Biology, vol. 73, no. 10, pp. 2249-2276, 2011.

[32] S. Bhattacharyya and D. K. Bhattacharya, "Pest control through viral disease: mathematical modeling and analysis," Journal of Theoretical Biology, vol. 238, no. 1, pp. 177-197, 2006.

[33] S. M. Moore, E. T. Borer, and P. R. Hosseini, "Predators indirectly control vector-borne disease: linking predator-prey and host-pathogen models," Journal of the Royal Society Interface, vol. 7, no. 42, pp. 161-176, 2009.

[34] K. W. Okamoto and P. Amarasekare, "The biological control of disease vectors," Journal of Theoretical Biology, vol. 309, pp. 4757, 2012.

[35] F. Y. Zhou and H.X. Yao, "Dynamics and biocontrol: the indirect effects of a predator population on a host-vector disease model," Abstract and Applied Analysis, vol. 2014, Article ID 252718, 14 pages, 2014.
[36] O. Diekmann, J. A. P. Heesterbeek, and J. A. J. Metz, "On the definition and the computation of the basic reproduction ratio $R_{0}$ in models for infectious diseases in heterogeneous populations," Journal of Mathematical Biology, vol. 28, no. 4, pp. 365-382, 1990.

[37] P. van den Driessche and J. Watmough, "Reproduction numbers and sub-threshold endemic equilibria for compartmental models of disease transmission," Mathematical Biosciences, vol. 180, pp. 29-48, 2002. 


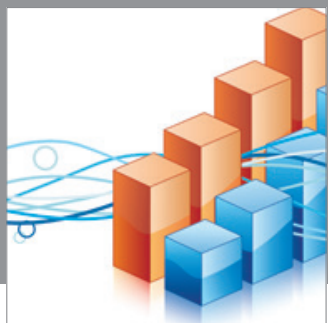

Advances in

Operations Research

mansans

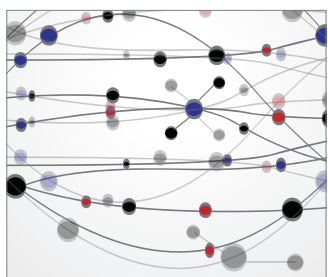

The Scientific World Journal
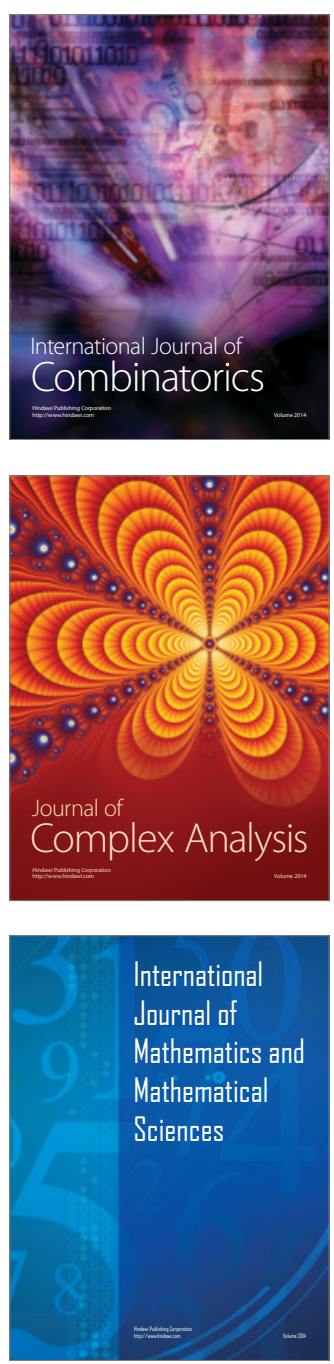
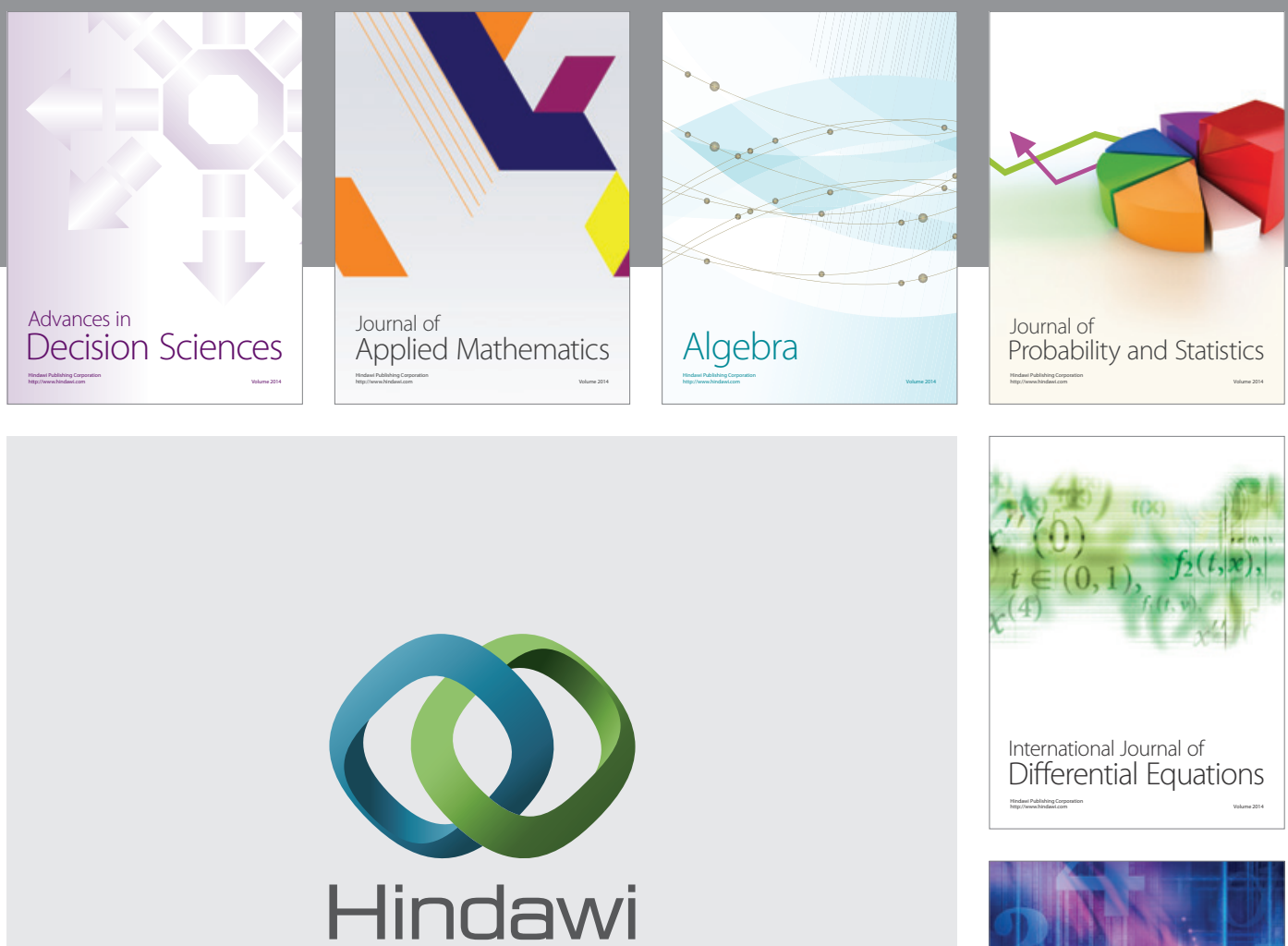

Submit your manuscripts at http://www.hindawi.com
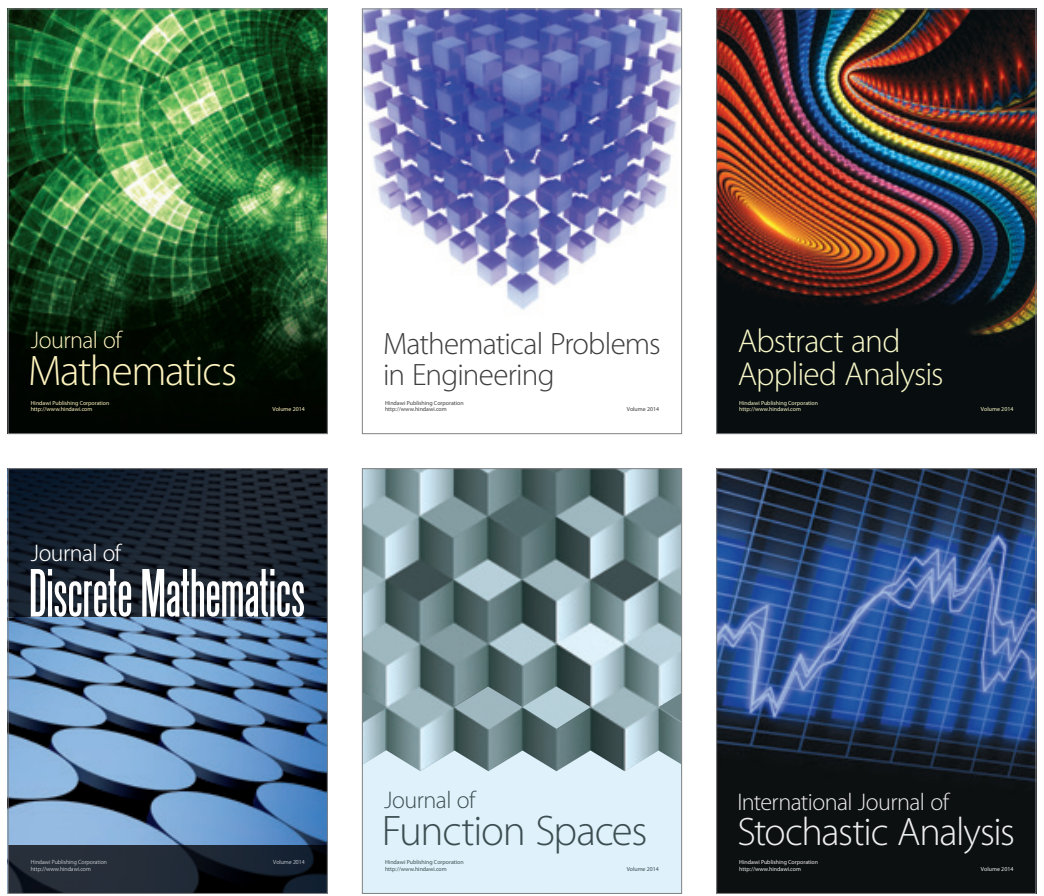

Journal of

Function Spaces

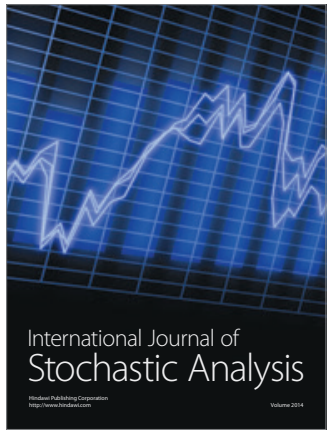

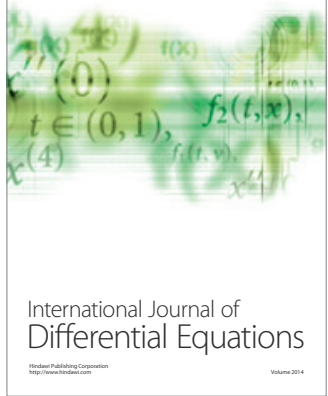
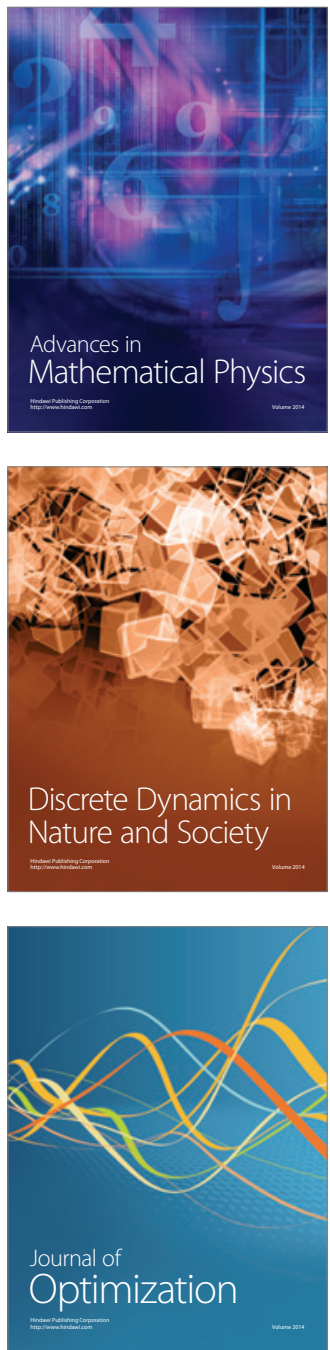